\title{
Non-Abelian linear Boltzmann equation and quantum correction to Kramers and Smoluchowski equation
}

\author{
Bassano Vacchini \\ Dipartimento di Fisica dell'Università di Milano and INFN, Sezione di Milano, Via Celoria 16, I-20133 Milan, Italy
}

(Received 22 January 2002; published 30 August 2002)

\begin{abstract}
A quantum linear Boltzmann equation, constructed in terms of the operator-valued dynamic structure factor of the macroscopic system the test particle is interacting with, is proposed. Due to this operator structure it is a non-Abelian linear Boltzmann equation and when expressed through the Wigner function it allows for a direct comparison with the classical one. Considering a Brownian particle, the corresponding Fokker-Planck equation is obtained in a most direct way taking the limit of small energy and momentum transfer. A typical quantum correction to the Kramers equation thus appears, describing diffusion in position and further implying a correction to Einstein's diffusion coefficient in the high temperature and friction limit in which the Smoluchowski equation emerges.
\end{abstract}

DOI: 10.1103/PhysRevE.66.027107

PACS number(s): 05.60.Gg, 05.30.-d, 05.40.Jc, 03.65.Yz

The study of irreversible processes, even in relatively simple cases in which one considers the reduced dynamics of a system with few degrees of freedom interacting with a suitable reservoir, is a subject of major interest in both classical and quantum mechanics. In the specific case of a microsystem whose interaction with a macroscopic system or reservoir can be described in terms of collisions, a major tool for the classical description of its dynamics is the linear Boltzmann equation (LBE) or transport equation. This equation has proved essential in fields like neutron transport theory [1] and is still the standard reference for this kind of dynamics even at quantum level [2], the only improvement being the introduction of the quantum collision cross section in place of the classical one. This kinetic equation, provided a suitable expansion of the collision term can be performed, then leads to a Fokker-Planck (FP) equation for the study of dissipation, which for Brownian motion (BM) is the so-called Kramers equation (KE). Considering situations in which dissipation is very important, $\mathrm{KE}$ in turn leads to the so-called Smoluchowski equation in which Einstein's diffusion coefficient appears.

In this paper, we give the structure of a fully quantum, non-Abelian LBE, non-Abelian just due to the presence in it of the operator-valued rather than $c$-number dynamic structure factor (DSF) of the macroscopic system, as typical of the quantum realm, giving a physical example of a recently obtained mathematical result on Lindblad generators of translation-covariant completely positive quantum dynamical semigroups [3]. Considering the BM of a test particle in a gas, a most straightforward expansion of the non-Abelian $\mathrm{LBE}$, written in terms of the Wigner function, leads to a $\mathrm{KE}$ with a typical quantum correction linked to position diffusion vanishing in the semiclassical limit $\hbar \rightarrow 0$. Such a correction ensures positivity at quantum level and is connected to the time evolution of the off-diagonal matrix elements of the statistical operator in the momentum representation, which can only be given in a fully quantum mechanical version of the LBE. Studying the high friction limit of this FP equation analogously to the classical case, a quantum correction to Einstein's diffusion coefficient also appears.

\section{NON-ABELIAN LINEAR BOLTZMANN EQUATION}

Let us start writing the LBE for the classical case, still relying on the quantum expression for the collision cross section, in a way that is actually not the most common one. According to Van Hove [4], the energy-dependent differential cross section per target particle describing scattering of a microscopic probe off a macroscopic sample is given by

$$
\frac{d^{2} \sigma}{d \Omega_{p^{\prime}} d E_{p^{\prime}}}=\frac{p^{\prime}}{p} \Sigma(q) S(q, E)
$$

if the momentum of the microscopic probe changes from $p$ to $\boldsymbol{p}^{\prime}=\boldsymbol{p}+\boldsymbol{q} . \quad \Sigma(q)$ is here the collision cross section for the single scattering event, given by $\Sigma(q)$ $=M^{2}(2 \pi \hbar)^{3}(2 \pi / \hbar)|\tilde{t}(q)|^{2}$, with $\tilde{t}(q)$ the Fourier transform of the $T$ matrix describing the one-pair collisions between the test particle and the particles constituting the sample, supposed to depend only on the modulus of the momentum transfer $\boldsymbol{q} . S(\boldsymbol{q}, E)$ is a two-point correlation function known as DSF [5], reflecting the equilibrium many-body properties of the fluid, depending on momentum $q$ and energy $E=E(\boldsymbol{q}, \boldsymbol{p})=q^{2} / 2 M+\boldsymbol{p} \cdot \boldsymbol{q} / M$ transferred to the test particle of mass $M$, whose general expression is given by the Fourier transform with respect to energy and momentum transfer of the time-dependent spatial autocorrelation function according to

$$
\begin{aligned}
S(\boldsymbol{q}, E)= & \frac{1}{2 \pi \hbar} \int d t \int d^{3} \boldsymbol{x} e^{i / \hbar(E t-\boldsymbol{q} \cdot \boldsymbol{x})} \\
& \times \frac{1}{N} \int d^{3} \boldsymbol{y}\langle N(\boldsymbol{y}) N(\boldsymbol{x}+\boldsymbol{y}, t)\rangle .
\end{aligned}
$$

Using Eq. (1) the LBE in the absence of external potentials may be written as [1]

$$
\begin{aligned}
\frac{\partial}{\partial t} f(\boldsymbol{x}, \boldsymbol{p}, t)= & -\frac{\boldsymbol{p}}{M} \cdot \boldsymbol{\nabla}_{x} f(\boldsymbol{x}, \boldsymbol{p}, t)+\frac{n}{M^{2}} \int d^{3} \boldsymbol{q} \Sigma(q) \\
& \times[S(\boldsymbol{q}, \boldsymbol{p}-\boldsymbol{q}) f(\boldsymbol{x}, \boldsymbol{p}-\boldsymbol{q}, t)-S(\boldsymbol{q}, \boldsymbol{p}) f(\boldsymbol{x}, \boldsymbol{p}, t)]
\end{aligned}
$$


with $S(\boldsymbol{q}, \boldsymbol{p}) \equiv S(\boldsymbol{q}, E)$ the DSF of the homogeneous macroscopic fluid of density $n$ the particle is interacting with. So far the classical case, all expressions appearing in Eq. (2) are $c$-number functions.

Recent work on the study of subdynamics in nonrelativistic quantum field theory [6] has led to a scattering theory derivation of a particular structure of master equation [7-9], which goes beyond the usual limitation of linear coupling and is a natural candidate to be considered as a fully quantum LBE. Instead of an equation for the classical distribution function it is an equation for the full statistical operator $\hat{\rho}$ associated to the test particle, describing not only its momentum distribution, but also its coherence properties. These are particularly relevant for the time evolution of the offdiagonal matrix elements, related to the phenomenon of decoherence, and for the low temperature behavior, where the classical particle viewpoint is insufficient and wave effects are to be taken into account. The quantum kinetic equation is given by

$$
\begin{aligned}
& \frac{d \hat{\rho}}{d t}=-\frac{i}{\hbar}\left[\hat{\mathrm{H}}_{0}, \hat{\rho}\right]+\frac{n}{M^{2}} \int d^{3} \boldsymbol{q} \Sigma(q) \\
& \times\left[e^{(i / \hbar) \boldsymbol{q} \cdot \hat{x}} \sqrt{S(\boldsymbol{q}, \hat{\mathrm{p}})} \hat{\rho} \sqrt{S(\boldsymbol{q}, \hat{\mathrm{p}})} e^{-(i / \hbar) \boldsymbol{q} \cdot \hat{\mathrm{x}}}\right. \\
& \left.-\frac{1}{2}\{S(\boldsymbol{q}, \hat{\mathrm{p}}), \hat{\rho}\}\right],
\end{aligned}
$$

with $\hat{\mathrm{x}}$ and $\hat{\mathrm{p}}$ position and momentum operator for the test particle, $\hat{\mathrm{H}}_{0}=\hat{\mathrm{p}}^{2} / 2 M, S(\boldsymbol{q}, \hat{\mathrm{p}})$ the operator-valued DSF, which is a positive operator due to Eq. (1). Equation (3) addresses the same physics as the LBE but is non-Abelian due to the appearance in it of operators instead of $c$-number functions. It provides a physical realization of the general mathematical structure of translation-covariant quantum dynamical semigroup [3], while other known physical examples are restricted to the diffusive case. Neglecting the dependence on the momentum operator $\hat{p}$ and therefore on the energy transfer of the DSF in (3), so that its appearance only corrects the integration measure, one recovers a structure of master equation typically proposed on a more phenomenological basis for the study of the phenomenon of decoherence, which has gone through many refinements [10], all missing the correct description of momentum and energy transfer, which may not be critical for the description of short-time decoherence but are crucial for the approach to the correct stationary state. In fact, while the models in Ref. [10] predict a steady growth of kinetic energy and do not admit a stationary state, provided the fluid is in a $\beta$-KMS (Kubo, Martin, Schwinger) state or equivalently the DSF satisfies the detailed balance condition, Eq. (3) admits a stationary solution of the form $\exp \left(-\beta \hat{p}^{2} / 2 M\right)$, with $M$ the mass of the test particle and $\beta$ the inverse temperature of the fluid [11]. In order to clarify analogies and differences between Eq. (2) and Eq. (3) we will now consider a dynamics in which also the off-diagonal elements of the statistical operator are of relevance, corresponding to position diffusion. We focus on a system in which the DSF can be explicitly calculated, a free gas of particles of mass $m$ satisfying MaxwellBoltzmann statistics (for the extension to quantum statistics see [11]), the expression of the correlation function being in this case incidentally the same for both classical and quantum realm [5]

$$
S_{\mathrm{MB}}(\boldsymbol{q}, \boldsymbol{p})=\sqrt{\frac{\beta m}{2 \pi}} \frac{1}{q} \exp \left[-\frac{\beta}{8 m} \frac{\left(2 m E(\boldsymbol{q}, \boldsymbol{p})+q^{2}\right)^{2}}{q^{2}}\right] .
$$

\section{QUANTUM CORRECTION TO KRAMERS EQUATION}

As a first step we recover from Eq. (2) the classical KE for the $\mathrm{BM}$ of a massive test particle in a gas of much lighter ones. Setting $\alpha=m / M$ the ratio between the masses and considering the Brownian limit of small $\alpha$, corresponding to small energy transfer, the correct limiting expression of Eq. (4) still satisfying the detailed balance condition is [11]

$$
S_{\mathrm{MB}}^{\infty}(\boldsymbol{q}, \boldsymbol{p})=\sqrt{\frac{\beta m}{2 \pi}} \frac{1}{q} e^{-(\beta / 8 m) q^{2}} e^{-(\beta / 2) E(\boldsymbol{q}, \boldsymbol{p})} .
$$

Starting from the LBE written in terms of the DSF as in Eq. (2) and using Eq. (5) one obtains in a most straightforward way KE. Substituting Eq. (5) in Eq. (2) one in fact has

$$
\begin{aligned}
\frac{\partial}{\partial t} f(\boldsymbol{x}, \boldsymbol{p}, t)= & -\frac{\boldsymbol{p}}{M} \cdot \nabla_{x} f(\boldsymbol{x}, \boldsymbol{p}, t)+\frac{n}{M^{2}} \sqrt{\frac{\beta m}{2 \pi}} \int d^{3} \boldsymbol{q} \frac{\sum(q)}{q} \\
& \times e^{-(\beta / 8 m)(1+2 \alpha) q^{2}}\left[e^{\left.-\boldsymbol{q} \cdot \nabla_{p}-1\right]}\right. \\
& \times e^{-(\beta / 2 M) \boldsymbol{q} \cdot \boldsymbol{p}} f(\boldsymbol{x}, \boldsymbol{p}, t)
\end{aligned}
$$

and considering the limit of small momentum transfer $\boldsymbol{q}$, expanding the exponentials and keeping terms up to second order one immediately has for an isotropic medium

$$
\begin{aligned}
\frac{\partial}{\partial t} f(\boldsymbol{x}, \boldsymbol{p}, t)= & -\frac{\boldsymbol{p}}{M} \cdot \nabla_{x} f(\boldsymbol{x}, \boldsymbol{p}, t) \\
& +\frac{n \beta}{6 M^{3}} \sqrt{\frac{\beta m}{2 \pi}} \int d^{3} \boldsymbol{q} q \sum(q) e^{-(\beta / 8 m)(1+2 \alpha) q^{2}} \\
& \times \sum_{i=1}^{3} \frac{\partial}{\partial p_{i}}\left[\left(p_{i}+\frac{M}{\beta} \frac{\partial}{\partial p_{i}}\right) f(\boldsymbol{x}, \boldsymbol{p}, t)\right]
\end{aligned}
$$

and therefore KE for the description of BM

$$
\begin{aligned}
(\partial / \partial t) f(\boldsymbol{x}, \boldsymbol{p}, t)= & -(\boldsymbol{p} / M) \cdot \boldsymbol{\nabla}_{x} f(\boldsymbol{x}, \boldsymbol{p}, t)+\eta\left\{\boldsymbol{\nabla}_{p} \cdot[\boldsymbol{p} f(\boldsymbol{x}, \boldsymbol{p}, t)]\right. \\
& \left.+(M / \beta) \boldsymbol{\Delta}_{p} f(\boldsymbol{x}, \boldsymbol{p}, t)\right\},
\end{aligned}
$$

with a friction coefficient explicitly given by

$$
\eta=\frac{1}{6} \frac{n}{M^{3}} \beta \sqrt{\frac{\beta m}{2 \pi}} \int d^{3} \boldsymbol{q} q \Sigma(q) e^{-(\beta / 8 m)(1+2 \alpha) q^{2}} .
$$

We now want to obtain the corresponding result from the non-Abelian LBE (3). To do this it is particularly convenient to use the Wigner function, which even if it is not a welldefined probability density, well serves the purpose of comparing quantum versus classical equations. Introducing the Wigner function $f_{\rho}^{\mathrm{W}}(\boldsymbol{x}, \boldsymbol{p}, t)$, Eq. (3) may be rewritten for 
a Brownian particle in a Maxwell-Boltzmann gas, using Eq. (5), as

$$
\begin{aligned}
\frac{\partial}{\partial t} f_{\rho}^{\mathrm{W}}(\boldsymbol{x}, \boldsymbol{p}, t)= & -\frac{\boldsymbol{p}}{M} \cdot \nabla_{x} f_{\rho}^{\mathrm{W}}(\boldsymbol{x}, \boldsymbol{p}, t)+\frac{n}{M^{2}} \sqrt{\frac{\beta m}{2 \pi}} \int d^{3} \boldsymbol{q} \frac{\Sigma(q)}{q} \\
& \times e^{-(\beta / 8 m)(1+2 \alpha) q^{2}}\left[e^{\left.-\boldsymbol{q} \cdot \boldsymbol{\nabla}_{p}-\cos \left(\frac{\beta \hbar}{4 M} \boldsymbol{q} \cdot \nabla_{x}\right)\right]}\right. \\
& \times e^{-(\beta / 2 M) \boldsymbol{q} \cdot \boldsymbol{p}} f_{\rho}^{\mathrm{W}}(\boldsymbol{x}, \boldsymbol{p}, t) .
\end{aligned}
$$

The comparison between Eq. (6) and Eq. (8) is straightforward: the cosine term in Eq. (8), arising from the nonAbelian structure of Eq. (3), is replaced by a factor 1 in the classical case, and this result can be simply obtained by taking the semiclassical limit $\hbar \rightarrow 0$ in Eq. (8), the argument of the cosine depending linearly on $\hbar$. The quantum correction to KE then comes from this contribution and amounts to a term corresponding to diffusion in position, as can be seen considering the small momentum transfer limit of Eq. (8)

$$
\begin{aligned}
\frac{\partial}{\partial t} f_{\rho}^{\mathrm{W}}(\boldsymbol{x}, \boldsymbol{p}, t)= & -\frac{\boldsymbol{p}}{M} \cdot \boldsymbol{\nabla}_{x} f_{\rho}^{\mathrm{W}}(\boldsymbol{x}, \boldsymbol{p}, t)+\eta\left[\boldsymbol{\nabla}_{p} \cdot\left[\boldsymbol{p} f_{\rho}^{\mathrm{W}}(\boldsymbol{x}, \boldsymbol{p}, t)\right]\right. \\
& \left.+\frac{M}{\beta} \boldsymbol{\Delta}_{p} f_{\rho}^{\mathrm{W}}(\boldsymbol{x}, \boldsymbol{p}, t)+\frac{\beta}{M} \frac{\hbar^{2}}{16} \boldsymbol{\Delta}_{x} f_{\rho}^{\mathrm{W}}(\boldsymbol{x}, \boldsymbol{p}, t)\right],
\end{aligned}
$$

with $\eta$ as in Eq. (7). Thus position and momentum diffusion do appear together in the quantum description of BM, even though with different weights, as appears from the different $\beta$ dependence of the two contributions, while the additional diffusion term vanishes in the limit $\hbar \rightarrow 0$. This can be seen more clearly by introducing the thermal momentum spread $\Delta p_{\mathrm{th}}^{2}=M / \beta$ and the thermal position spread or square thermal wavelength $\Delta x_{\mathrm{th}}^{2}=\beta \hbar^{2} / 4 M$, satisfying the minimum uncertainty relation $\Delta p_{\mathrm{th}} \Delta x_{\mathrm{th}}=\hbar / 2$, so that one has

$$
\begin{aligned}
(\partial / \partial t) f_{\rho}^{\mathrm{W}}= & -(\boldsymbol{p} / M) \cdot \boldsymbol{\nabla}_{x} f_{\rho}^{\mathrm{W}}+\eta\left[\boldsymbol{\nabla}_{p} \cdot\left(\boldsymbol{p} f_{\rho}^{\mathrm{W}}\right)+\Delta p_{\mathrm{th}}^{2} \boldsymbol{\Delta}_{p} f_{\rho}^{\mathrm{W}}\right. \\
& \left.+\frac{1}{4} \Delta x_{\mathrm{th}}^{2} \boldsymbol{\Delta}_{x} f_{\rho}^{\mathrm{W}}\right] .
\end{aligned}
$$

The FP equation (9) or Eq. (10) can be recast in operator form, leading to the well-known structure [12]

$$
\begin{aligned}
\frac{d \hat{\rho}}{d t}= & -\frac{i}{\hbar}\left[\hat{\mathrm{H}}_{0}, \hat{\rho}\right]-\frac{i}{\hbar} \frac{\eta}{2} \sum_{i=1}^{3}\left[\hat{\mathrm{x}}_{i},\left\{\hat{\mathrm{p}}_{i}, \hat{\rho}\right\}\right] \\
& -\frac{\eta}{\hbar^{2}} \Delta p_{\mathrm{th}}^{2} \sum_{i=1}^{3}\left[\hat{\mathrm{x}}_{i},\left[\hat{\mathrm{x}}_{i}, \hat{\rho}\right]\right]-\frac{\eta}{\hbar^{2}} \frac{1}{4} \Delta x_{\mathrm{th}}^{2} \sum_{i=1}^{3}\left[\hat{\mathrm{p}}_{i},\left[\hat{\mathrm{p}}_{i}, \hat{\rho}\right]\right],
\end{aligned}
$$

which could have been obtained directly from Eq. (3) as in [11], without going through the use of the Wigner function, missing however in this way the particularly manifest appearance of the quantum correction that provides an inhomogeneous contribution to the collision term in the LBE through the appearance of the cosine in Eq. (8). The appear- ance of both position and momentum diffusions in Eq. (10) or equivalently in Eq. (11) is due to the fact that Eq. (3) is an evolution equation not only for the diagonal elements of the statistical operator giving the momentum distribution of the test particle, but also for its quantum coherence properties, which actually require preservation of the positivity of the statistical operator. In fact the quantum correction in Eq. (10), actually corresponding to the double commutator with the momentum operator in Eq. (11), is necessary for Eq. (11) to have a Lindblad structure ensuring complete positivity (corresponding with positivity for FP equations of the form (11) [13]). Correctness and relevance of this term are heavily debated $[14,7,8,11]$, and its appearance as a typical quantum correction coming from the Brownian and small momentum transfer limit of the non-Abelian LBE (3) expressed in terms of the operator-valued DSF sheds light on its physical origin. The quantum FP equation (11) due to the minimum uncertainty relation $\Delta p_{\text {th }} \Delta x_{\text {th }}=\hbar / 2$ has furthermore the distinguishing feature of being expressible in terms of a single generator for Cartesian direction [7], a property actually required for the diffusive component of the generator of a translation-covariant semigroup [3]: both Eq. (3) and Eq. (11) are proper generators of completely positive quantum dynamical semigroups, admitting the correct equipartition stationary solution and being invariant under translations. The three features of complete positivity, equipartition, and covariance are in fact not contradictory provided one considers covariance under the symmetry relevant to the physical problem, which is not necessarily translation invariance, otherwise, independently of positivity or complete positivity of the time evolution, covariance would lead to high nonuniqueness of the stationary solution [15]. Equations (3) and (11) are essentially the two possible structures of generators of translation-covariant semigroups, the results exposed in [3] giving the quantum counterpart of the well-known classical result according to which the only meaningful approximation of the integro-differential LBE as a differential equation is a second order FP equation [16].

Now that the quantum correction to KE has been givenwhich unlike previous results is not dependent on the presence of external potentials [17], but only relates to Heisenberg's uncertainty principle [12]—we move on to investigate whether this correction also has consequences on the Smoluchowski equation (SE) for the description of BM in the limit of high temperature and friction, when momentum is expected to quickly relax to its equilibrium value and one is only interested in the time evolution of the slowly varying marginal position distribution $\sigma_{\rho}^{\mathrm{W}}(\boldsymbol{x}, t)=\int d^{3} \boldsymbol{p} f_{\rho}^{\mathrm{W}}(\boldsymbol{x}, \boldsymbol{p}, t)$.

\section{QUANTUM CORRECTION TO SMOLUCHOWSKI EQUATION}

We therefore start from Eq. (9), essentially following the derivation of the SE from the KE in the high friction limit given by van Kampen [18], though more refined derivations have been given [19], differing however only for higher order contributions. Setting

$$
\eta(\beta / M)\left(\hbar^{2} / 16\right)=(\eta / 4) \Delta x_{\mathrm{th}}^{2} \equiv D_{x x}
$$


and considering a high temperature, strong friction situation, in which the quantum position diffusion term can be considered as a perturbation, we write Eq. (9) as

$$
\boldsymbol{\nabla}_{p} \cdot\left(\boldsymbol{p} f_{\rho}^{\mathrm{W}}\right)+\frac{M}{\beta} \boldsymbol{\Delta}_{p} f_{\rho}^{\mathrm{W}}=\frac{1}{\eta}\left[\frac{\partial}{\partial t} f_{\rho}^{\mathrm{W}}+\frac{\boldsymbol{p}}{M} \cdot \boldsymbol{\nabla}_{x} f_{\rho}^{\mathrm{W}}-D_{x x} \boldsymbol{\Delta}_{x} f_{\rho}^{\mathrm{W}}\right] .
$$

Introducing the differential operators

$$
\begin{gathered}
K^{(0)}[g]=\nabla_{p} \cdot(\boldsymbol{p} g)+\frac{M}{\beta} \boldsymbol{\Delta}_{p} g \\
\frac{1}{\eta} L^{(1)}[g]=\frac{1}{\eta}\left[\frac{\partial}{\partial t} g+\frac{\boldsymbol{p}}{M} \cdot \nabla_{x} g-D_{x x} \boldsymbol{\Delta}_{x} g\right]
\end{gathered}
$$

and expressing $f_{\rho}^{\mathrm{W}}$ as an expansion in powers of $1 / \eta$

$$
f_{\rho}^{\mathrm{W}}=f_{\rho}^{\mathrm{W}(0)}+(1 / \eta) f_{\rho}^{\mathrm{W}(1)}+\cdots,
$$

Eq. (13) may be rewritten as $K^{(0)}\left[f_{\rho}^{\mathrm{W}}\right]=(1 / \eta) L^{(1)}\left[f_{\rho}^{\mathrm{W}}\right]$ and solved by iteration using Eq. (14) and equating on both sides contributions of the same order in $\eta$. The only difference with respect to the classical situation considered in Ref. [18] lies in the structure of the $L^{(1)}$ operator, where also the Laplacian with respect to position appears. Going through the very same procedure, integrating over the fast degree of freedom given by the momentum dependence of the Wigner distribution function, and neglecting terms higher than first order in $1 / \eta$ one obtains again for the marginal position distribution $\sigma_{\rho}^{\mathrm{W}}(\boldsymbol{x}, t)$ the $\mathrm{SE}$

$$
(\partial / \partial t) \sigma_{\rho}^{\mathrm{W}}(\boldsymbol{x}, t)=\left[(1 / \eta M \beta)+D_{x x}\right] \boldsymbol{\Delta}_{x} \sigma_{\rho}^{\mathrm{W}}(\boldsymbol{x}, t)
$$

with a diffusion coefficient which is however not simply Einstein's, but has a quantum correction that vanishes in the semiclassical limit $\hbar \rightarrow 0$, just given by the coefficient (12) responsible for position diffusion in the quantum KE (9). The new overall coefficient can be written as $1 /(\eta M \beta)[1$ $\left.+(\eta \beta \hbar)^{2} / 16\right]$, so that the correction is actually given by the square ratio between two characteristic times, $\beta \hbar$ for the bath and $1 / \eta$ for the test particle, which should be much less than that according to the Markov approximation.

We have thus proposed a fully quantum, non-Abelian LBE given by (3) and expressed in terms of the $T$ matrix describing collisions and the operator-valued DSF of the environment, thus going beyond the usual restriction of linear dissipation. Considering a massive test particle interacting with a gas of much lighter particles the LBE written in terms of the DSF leads in a most straightforward way to the KE for the description of BM in both classical and quantum cases. One simply has to consider the clear-cut physical limit in which momentum transfer and energy transfer (or equivalently ratio between the masses) are small, thus obtaining in the classical case KE and in the quantum case (9) with a peculiar, heavily debated quantum correction linked to position diffusion, whose appearance is to be traced back to the $\hbar$ dependent, inhomogeneous cosine correction in Eq. (8) vanishing in the semiclassical limit. Further studying the case of high temperature and friction the quantum SE (15) is obtained, in which Einstein's diffusion coefficient is slightly modified due to the presence of the quantum position diffusion coefficient (12), which depends linearly on the inverse temperature and vanishes if $\hbar \rightarrow 0$. The non-Abelian LBE (3) due to its intrinsic quantum structure should prove as a sound starting point for the study of quantum kinetic, in which not only the momentum distribution, but also coherence properties are of relevance; it, furthermore, has the advantage of being expressed in terms of quantities of direct physical meaning, such as the DSF and the collision cross section, for which suitable phenomenological ansatz or experimentally determined expressions can be inserted.
[1] M. M. R. Williams, The Slowing Down and Thermalization of Neutrons (North-Holland, Amsterdam, 1966).

[2] E. Timmermans and R. Côté, Phys. Rev. Lett. 80, 3419 (1998); G. Ferrari, Phys. Rev. A 59, R4125 (1999).

[3] A.S. Holevo, Rep. Math. Phys. 32, 211 (1993); 33, 95 (1993); J. Math. Phys. 37, 1812 (1996).

[4] L. van Hove, Phys. Rev. 95, 249 (1954).

[5] S.W. Lovesey, Theory of Neutron Scattering from Condensed Matter (Clarendon Press, Oxford, 1984).

[6] L. Lanz and B. Vacchini, Int. J. Mod. Phys. A 17, 435 (2002).

[7] B. Vacchini, Phys. Rev. Lett. 84, 1374 (2000).

[8] R.F. O'Connell, Phys. Rev. Lett. 87, 028901 (2001); B. Vacchini, ibid. 87, 028902 (2001).

[9] B. Vacchini, Phys. Rev. E 63, 066115 (2001).

[10] E. Joos and H.D. Zeh, Z. Phys. B: Condens. Matter 59, 223 (1985); M.R. Gallis and G.N. Fleming, Phys. Rev. A 42, 38 (1990); R. Alicki, ibid. 65, 034104 (2002).

[11] B. Vacchini, J. Math. Phys. 42, 4291 (2001).

[12] A. Isar, Fortschr. Phys. 47, 855 (1999).
[13] P. Talkner, Ann. Phys. (N.Y.) 167, 390 (1986).

[14] V. Ambegaokar, Ber. Bunsenges. Phys. Chem. 95, 400 (1991); L. Diósi, Europhys. Lett. 22, 1 (1993); 30, 63 (1995); W.J. Munro and C.W. Gardiner, Phys. Rev. A 53, 2633 (1996); S. Gao, Phys. Rev. Lett. 79, 3101 (1997); H.M. Wiseman and W.J. Munro, ibid.. 80, 5702 (1998); S. Gao, ibid. 80, 5703 (1998); G.W. Ford and R.F. O’Connell, ibid. 82, 3376 (1999); S. Gao, ibid. 82, 3377 (1999).

[15] D. Kohen et al., J. Chem. Phys. 107, 5236 (1997); B. Vacchini, e-print quant-ph/0204071, J. Math. Phys. (to be published).

[16] R.F. Pawula, Phys. Rev. 162, 1861 (1967).

[17] S. Ishioka, J. Phys. Soc. Jpn. 48, 367 (1980); P. Pechukas, J. Ankerhold, and H. Grabert, Ann. Phys. (Leipzig) 9, 794 (2000); J. Ankerhold, P. Pechukas, and H. Grabert, Phys. Rev. Lett. 87, 086802 (2001).

[18] N.G. van Kampen, Stochastic Processes in Physics and Chemistry (North-Holland, Amsterdam, 1992).

[19] L. Bocquet, Am. J. Phys. 65, 140 (1997). 